


\title{
El libro de texto mal empleado: Andreas Capellanus y la escena inicial de La Celestina
}

\section{The Mishandled Text-Book: Andreas Capellanus and the Opening Scene of La Celestina}

\author{
Alan Deyermond $\dagger$
}

Este artículo estudia la presencia del libro De Amore de Andreas Capellanus en la escena inicial de La Celestina. A través del análisis del primer parlamento de Calisto y las respuestas que le da Melibea, Deyermond propone que el joven personaje está empleando la obra de Capellanus a modo de libro de texto que le serviría de guía para conquistar a la dama, pero fracasa al intentarlo. Se plantea dos preguntas respecto a esta situación: una en cuanto a la accesibilidad de De Amore en la Edad Media y otra que se refiere a la probabilidad del uso consciente de esta obra por parte del autor de La Celestina. Concluye que, si se asume positivamente la imitación consciente de la obra de Capellanus y el autor tuviera la intención de presentar un amante fracasado, entonces sería factible pensar en un Calisto que, tras previos encuentros con Melibea, estuviera intentando prepararse para la siguiente entrevista; lo que abriría posibilidades de un desarrollo cómico de La Celestina ante los ojos de lectores conocedores de De Amore.

Palabras Clave: De amore, amante fracasado, desarrollo cómico

This article studies the presence of De Amore by Andreas Capellanus in the opening scene of La Celestina. Through the analysis of Calisto's first parliament, and the answers given by Melibea, Deyermond proposes that Calisto is using De amore as a text-book that would serve him as a guide to conquer Melibea. However, he fails in doing it, since he mishandles the contents of the book. Two questions arise from this situation: one regarding the accessibility of De Amore during the Middle Ages, and the other which refers to a probable and conscious use of Capellanus work by the author of La Celestina. Deyermond concludes that, if there is a conscious imitation of De Amore, it is possible to assume that the author intended to present Calisto as a failed lover, trying to learn from Capellanus , and preparing his next encounter with Melibea. This, therefore, would open possibilities for a comic development of La Celestina before the eyes of scholars and readers who had knowledge of De Amore.

KeYwORDs: De Amore, unsuccessful lover, comic development 
$\mathrm{L}$ a interpretación de La Celestina, incluso su género, son asuntos de gran controversia, aunque al menos el marco principal de la historia es claro: Calisto, en un principio rechazado por Melibea, conquista su apasionado corazón con la ayuda de la vieja alcahueta Celestina. La pasión de ambos les trae la desgracia a ellos y a quienes los rodean, y la obra culmina en un ambiente de profunda tragedia. Al principio, empero, el tono es distinto. El parlamento inicial que Calisto le dirige a Melibea se lleva a cabo en términos rimbombantes, exagerados y — según resulta — ridículamente infructíferos. Las respuestas ambiguas de Melibea lo impulsan a continuar, hasta que la brutal desilusión final lo hace parar en seco, se enreda más y más en su retórica amatoria. Nos podemos preguntar por qué Calisto esperaba que esta táctica tuviera éxito; la respuesta, a mi parecer, es que ésta es la táctica que su 52 libro de texto le dijo que adoptara.

Hoy en día podemos mencionar una variedad de obras teóricas sobre el amor cortés, pero los hombres y mujeres medievales tenían sólo un tratado importante en el cual confiar: el De Amore libri tres de Andreas Capellanus, que se escribió probablemente en Troyes a finales del siglo XII, a inspiración de María, la Condesa de Champaña. ${ }^{1}$ La división de la obra, como lo han hecho notar sus editores, es similar a la de Ovidio: El libro I trata de la conquista del amor, el libro II de su preservación ( $c f$. Ars Amatoria I y II), mientras que el libro III es "De reprobatione amoris" ( $c f$. Remedium amoris de Ovidio). El libro I, que es sustancialmente más largo que los otros dos juntos, sería obviamente el más atractivo para un hombre en la situación de Calisto, en especial porque contiene una variedad de diálogos prototípicos entre hombres y mujeres de varias clases sociales. Si un amante irresoluto no sabía cómo dirigirse a su amada, Andreas estaba a la mano para guiarlo. La sujeción de Calisto a las tácticas de Andreas puede observarse con la comparación de sus comentarios iniciales y las primeras oraciones del hombre en tres de los diálogos de De Amore:

${ }^{1}$ Texto latino editado por E. Trojel (1892); con traducción catalana editada por A. Pagès (1930). Traducción J.J. Parry (1941). Cf. A. J. Denomy (1947), A. Kelly (1937) y C. S. Lewis (1936: 32-43). 
[Loquitur plebeius ad plebeiam]

Quando te divina formavit essentia, nulla sibi alia facienda restabant: Tuo

Calisto. En esto veo, Melibea, la grandeza de Dios.

decori nihil deesse cognosco...

[Loquitur nobilior plebeiae]

Melibea. ¿En que, Calisto?

Calisto. En dar poder a natura que de

A longinquis retro temporibus diem istam desidaveri et plenarie in mente gessi propositum maem bobis aperire mentem

et intentionem et, quanta mihi sit de vobis assidue cogitatio. Temporis tamen inopportunitas usque nunc distulit amantis eloquium.

[Loquitur nobilior nobili]

Maiores mihi restant Deo gratiae referendae quam cuiquam in orbe viventi, quia hoc, quod meus animus

videre super omnia cupiebat, nunc corporali mihi visu est concessum aspicere, et hoc mihi Deum credo praemium concessisse propter nimium desiderii mei affectum, et quia mei voluit exautan perfecta fermosura te dotasse, $y$ fazer a mi inmérito tanta merced que verte alcançasse, y en tan conueniente lugar,

mi secreto dolor manifestarte pudiesse. Sin dubda, incomparable- mente es mayor tal galardón que el seruicio, sacrificio, deuoción y obras pias que por este lugar alcançar yo tengo a Dios offrecido. ¿Quién vido en esta vida cuerpo glorificado de ningún hombre como agora el mío? Por cierto, los gloriosos santos, que se deleytan

en la visión diuina, no gozan mas que yo agora en el acatamiento tuyo.

(Rojas 1958: 23)

dire preces importune precantis. Non enim poterat diei vel noctis hora pertransire continua, qua Deum non exorarem attentius, ut corporaliter vos ex propinquo vivendi mihi concederet largitatem

(Trojel 1892:21, 110-111, 124-125, Pagès 1930: 11, 64, 73-74, Parry 1941: 37, 84, 92 )

Evidentemente esto no es una traducción directa del texto latino de Andreas, pero el parecido es incuestionable, en particular cuando llegamos al tercer extracto que (dado que viene de un diálogo entre un hombre y una mujer de diferentes grados de nobleza) podría bien haber sido el primero en atraer la atención de Calisto.

No obstante, si ha de aceptarse que Calisto se basaba en Andreas, dos preguntas tienen que resolverse: una tiene que ver con la historia literaria y 
la otra con la trama de La Celestina. La primera es: ¿era De Amore asequible para el autor de esta escena? De Amore parece haber tenido amplia circulación en la Edad Media y su popularidad continuó incluso hasta el siglo XVII. Doce manuscritos, más algunos fragmentos, todavía se conservan (Parry 1941: 2224). Fue impreso desde una fecha temprana, quizá tan temprana como 1473 o 1474 ( ¿en Estrasburgo?). Antes de la composición de La Celestina, habían existido dos traducciones al francés, dos al italiano y dos al alemán (una de las versiones al alemán se imprimió en tres ocasiones en el siglo Xv). Es aún más significativo que se realizara una traducción al catalán a finales del siglo XIV, relacionada con las Cortes de Amor amparadas bajo los auspicios del rey Juan I de Aragón y la reina Violante de Bar (Pagès 1930: xiv-xvi; Parry 1941: 23). La influencia de Andreas en la literatura española nunca se ha estudiado 54 de manera sistemática, pero de la poca información disponible podemos deducir que De Amore debió haber sido un texto conocido al menos en algunos círculos. Pagès (1930: xvi-xxxi), basándose parcialmente en investigaciones previas, ha trazado la influencia de De Amore en la poesía catalana desde el Faula de Guillem Torroella (ca. 1381) a la Gloria d'amor de Fra Rocaberti (ca. 1461). En Castilla, el Corbacho (de 1438) del Arcipreste de Talavera se basa en gran parte en el tercer libro de Andreas (Martínez de Toledo 1955: xx-Xxx, 229-239), y Juan Rodríguez del Padrón, en su Cadira del honor (del segundo cuarto del siglo Xv), cita a Andreas entre algunos otros personajes de autoridad (1884: 137). El prestigio y la difusión de De Amore fueron tales que casi con seguridad habría sido conocido por un estudiante universitario español de la segunda mitad del siglo Xv; y ya sea que el acto I sea atribuido a Fernando de Rojas, que era estudiante en Salamanca, o, tal como Anna Krause ha sugerido plausiblemente (1953), a un estudiante y compañero de Rojas, de quien él tomó la obra y la completó, no hay nada que debiera sorprendernos de la información sobre De Amore que aquí se revela.

La otra pregunta que surge tiene que ver con la probabilidad, y las implicaciones, de un uso consciente de los diálogos de Andreas (que se emplearon, ya sea de manera consciente o no, se demuestra, me parece, con lo anterior). Por supuesto, no podemos descartar la posibilidad de una reminiscencia inconsciente, aunque el uso de las oraciones iniciales de estos tres diálogos y la concordancia general en la organización de las ideas entre Andreas y La Celestina parecen inclinar la balanza en contra de esta posibilidad. Tampoco podemos descartar la posibilidad de una fuente intermedia, que se desprende de Andreas y que fue empleada por el autor del aucto I; aunque no he encontrado hasta ahora tal fuente. Sin embargo, si estamos frente a una imitación consciente de De Amore, ¿cuál es el propósito que buscaba el autor al 
incluirla? En general se ha aceptado que la escena inicial de La Celestina es el primer encuentro entre Calisto y Melibea, pero hay muchas evidencias en el texto para creer que el autor quería que nosotros sospecháramos de un encuentro previo entre la pareja. Si es así, y si Calisto se nos presenta hasta aquí como un amante fracasado, es tal vez legítimo imaginarlo preparándose para su esperado encuentro con Melibea buscando discursos adecuados en De Amore. Seguramente esto sería acorde con su carácter: en el aucto I, más que en el resto de La Celestina, se le muestra como un amante tonto y pedante. Al ser el tipo de persona que es, actúa tal y como le dice el libro, y su desesperación es por lo tanto aún mayor cuando no logra obtener la resolución del libro de texto. Melibea lo rechaza con extrema violencia; desesperado, él recurre a Celestina, y se inicia el camino hacia la sombría tragedia en la que Rojas convierte la obra. Pero la tragedia y la comedia pueden coexistir, y si el autor del aucto I es un estudiante que escribe en principio para gente como él, esta presentación del héroe que es desilusionado inesperadamente por una persona de autoridad, abre considerables posibilidades de un desarrollo cómico para los lectores que, como Calisto, conocen De Amore. 
